(C) 2018 IEEE. Personal use of this material is permitted. Permission from IEEE must be obtained for all other uses, in any current or future media, including reprinting/republishing this material for advertising or promotional purposes, creating new collective works, for resale or redistribution to servers or lists, or reuse of any copyrighted component of this work in other works

This is the accepted manuscript of: Alessandro Mingotti, Lorenzo Peretto, Roberto Tinarelli, "Low Power Voltage Transformer Accuracy Class Effects on the Residual Voltage Measurement," 2018 IEEE International Instrumentation and Measurement Technology Conference (I2MTC), Houston, TX, USA, 2018, pp. 1-6, doi: 10.1109/I2MTC.2018.8409807 


\title{
Low Power Voltage Transformer Accuracy Class Effects on the Residual Voltage Measurement
}

\author{
Alessandro Mingotti, Lorenzo Peretto, Roberto Tinarelli \\ Department of Electrical, Electronic and Information Engineering "G. Marconi” \\ Alma Mater Studiorum - University of Bologna \\ Bologna, Italy
}

\begin{abstract}
The paper addresses one of the new and most important issues arising when Low Power Voltage Transformers (LPVT) are used in power network substations for evaluating, among others, also the residual voltage. Conversely to opentriangle inductive instrument transformers, the use of phase voltage transformers for measuring the residual voltage gets challenging due to the very high accuracy required for the three LPVTs. In the paper, an analytical study will be presented along with simulation results in order to show the relationship between the accuracy class and the uncertainty affecting the residual voltage value.
\end{abstract}

Keywords - Voltage Transformer; Residual Voltage; Simulation; Low Power Instrument Transformer; Power Transformer; Accuracy Class; Voltage Error;

\section{INTRODUCTION}

The decarbonisation of energy systems has been a political decision taken in the recent years by all most industrialized countries in the world. European Commission's energy and climate policy, such as the SET-Plan, foresees that the context of the future scenarios for electric power networks will be that of ensuring a stable and secure power supply as Renewable Energy Sources (RES) penetration increases up to $100 \%$.

As a matter of fact, the wide-scale introduction of decentralized RES is causing significant and unprecedented changes in electrical power grids. Consequently, future electrical power grids will require real-time capable control and monitoring systems to ensure stability under increasingly complex and challenging conditions.

The generation of analogue measurement and control systems in power grid substations are approaching the end of their useful lifespan. More often their replacement is based on digital substation automation solutions according to IEC 61850 [1] and the use of new technologies to perform in a more efficient way distribution (like the massive use of Ring Main Units), measurements and control of the power network operation.

More specifically, Instrument Transformers (ITs) [2-4] are experiencing special attention by Utilities and private customers for different important reasons. Among the most important ones, with the event of Smart Grids and Distributed Energy Resources (DER) new performance and features are requested to the IT in order to accomplish real-time network control with the highest efficiency, speed and accuracy. For instance, the large use of power inverters for interconnecting large photovoltaic plants to

This research has been partially funded by the European project Horizon H2020 "ADMS: Smart Grid Active Distribution Management System to accommodate Renewable Energy Sources and Low Carbon Emissions". the grid has led to the injection of high order harmonics, which can interfere with industrial frequency component or among them to give rise to intermodulation. All this needs that such spectral components be correctly and accurately measured in order to let such systems to run under real-time feedback control. Moreover, in case of off-nominal frequency, protection relays must now trip faster than before (in few ms, instead of tens of $\mathrm{ms}$ as in the past). Furthermore, given that energy is no more flowing in just one direction (multiple producers or prosumers are now inter-connected to the same grid), very accurate energy and power measurements must be performed in order to correctly split the energy production revenues among them and for a proper accurate reactive energy injection into the grid. Again, the mass deployment of secondary substations and measurement nodes have also yield to have space and size constraints. Therefore, new requests for reduced dimensions of all electrical apparatus and systems have become a key parameter. Last but not least, the development and diffusion of digital communications between different nodes of the power network have requested for the ITs, as stated before, to feature also digital outputs.

At the light of all this, one of the most important measurements for network stability and diagnostic still remains the residual voltage. Its measurement is mandatory for protection coordination, like the implementation of differential protections (fault upstream or downstream the measurement node), to correctly classify ground faults, etc. Normally such a measurement was and is presently performed by using inductive voltage transformers star-connected at the primary-side and with open triangle at the secondary side. Typical accuracies required for the residual voltage measurements are in the order of some percent, while residual voltage values are in the order of few percent (typical value is $4 \%$ ).

In case the residual voltage is evaluated by using the line voltages (hence it is evaluated by summing the three secondary voltages of the voltage ITs), reaching such an accuracy in its measurement (which, we remember, is few percent of the rated voltage) gets very challenging. This is what is requested to the new devices used as voltage sensors, the Low Power Voltage Transformers (LPVT). Usually such devices reduce the phaseto-ground primary voltage to amplitudes of few volts. The secondary voltage is referred to ground as well. So, when using LPVTs in a three-phase system, the residual voltage must be evaluated by summing the three phase voltages. In such a case matching the existing requirements in terms of accuracy of the residual voltage measurement require for the LPITs to feature 


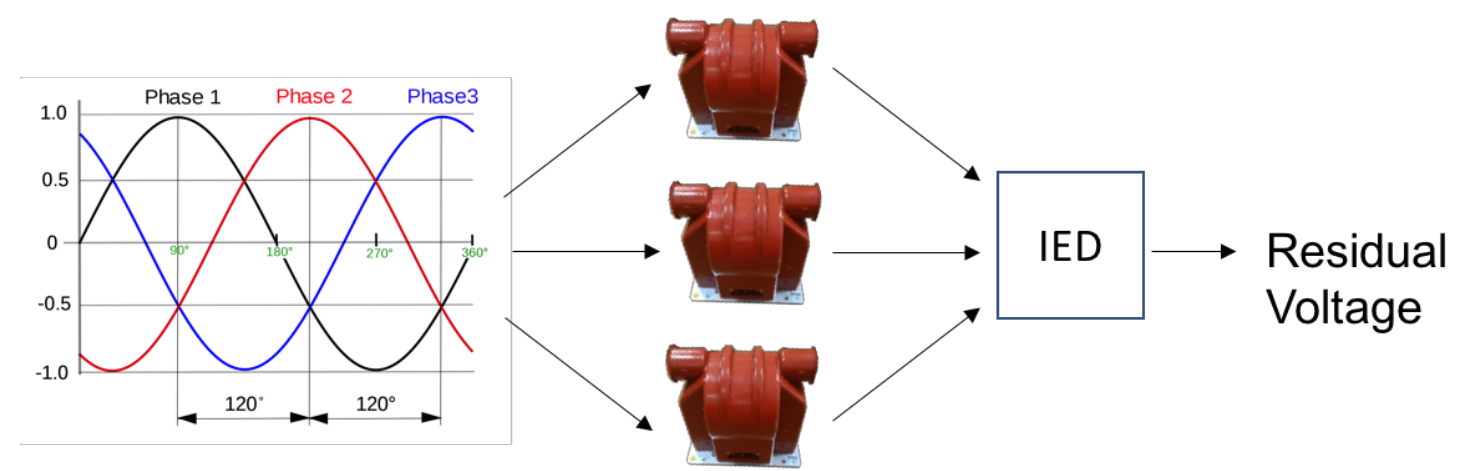

Fig. 1. Schematic of a typical setup for the measurement of three-phase system of symmetric voltages

special accuracy classes (far better than those of the inductive voltage transformers).

The paper is aimed at presenting the results of the study performed to correlate the uncertainty affecting the measurement of the residual voltage with the accuracy class of the LPVT. First of all, the maximum error that can occur when the residual voltage is computed as sum of the line-to-neutral voltages is determined. Then, an easy-to-use expression for estimating the uncertainty on the residual voltage is derived. It will be shown that it provides accurate results without the use of complex and long calculation as it would be required by the application of analytical or numerical methods suggested by the GUM [5] as well as its Supplement 1 [6]

Such a study is requested by industry and for completing what is presently reported in the new product Standard on LPVT IEC 61869-11, [7], and in future IEC 61869-105, [8], document dealing with uncertainty in calibration of ITs. Moreover, it will result useful to power network and system designers and operators for selecting suitable LPVTs according also to the accuracy requested for the residual voltage measurement.

The paper is structured as follows: in Section II the case study is defined and the mathematic development of the residual voltage, considering the VTs contributes, is presented. Section III contains the maximum error algorithm in order to find which combination of ratio and phase errors results in the maximum error associated to the residual voltage. In Section IV, the aforementioned simplified expression for the uncertainty evaluation is presented; finally, Section V summarize the presented study along with some conclusions and future works.

\section{Residual Voltage}

\section{A. Case-study definition}

According to the International Electrotechnical Vocabulary (IEV) the residual voltage $v_{r}(t)$ is defined as "the sum of the instantaneous values of all three line-to-hearth voltages, in a three-phase system" [9]:

$$
v_{r}(t)=v_{1}(t)+v_{2}(t)+v_{3}(t)
$$

where $v_{1}(t), v_{2}(t)$ and $v_{3}(t)$ are the instantaneous line to earth voltages of line 1,2 and 3 , respectively. In the case of a sinusoidal steady-state condition (1) turns into:

$$
\bar{V}_{R}=\bar{V}_{1}+\bar{V}_{2}+\bar{V}_{3}
$$

where $\bar{V}_{i}$ is the phasors of the generic quantity $v_{i}(t)$. Even if the residual voltage is a phasor (as in (2)) or a waveform (as in (1)), in practical application only its module is used.

As mentioned before, the paper presents the study oriented to show how the measurement of the residual voltage is affected by the accuracy class of the three LPVTs. Fig. 1 shows a typical setup for the measurement of such a quantity, referred to as $\bar{V}_{R}$. It consists in three LPVTs and an Intelligent Electronic Device (IED), which acquires the LPVTs outputs and computes the residual voltage. Therefore, the value attributed to $\bar{V}_{R}$ is affected by the effect of the uncertainty sources located in the LPVTs as well as in the IED. Usually, the latter can be considered negligible with respect to former one.

\section{B. Mathematic development}

At the light of this, let us consider three LPVTs featuring ratio errors $\varepsilon_{1}, \varepsilon_{2}$ and $\varepsilon_{3}$ and phase errors $\Delta \varphi_{1}, \Delta \varphi_{2}$, and $\Delta \varphi_{3}$ as defined in [4]. Therefore, starting from (2), the expression of the residual voltage, $\bar{V}_{R}$ can be expressed as a function of such uncertainty contributions:

$$
\begin{gathered}
\bar{V}_{R}=\left[V_{1}\left(1+\varepsilon_{1}\right) e^{j\left(\vartheta_{1}+\Delta \varphi_{1}\right)}+V_{2}\left(1+\varepsilon_{2}\right) e^{j\left(\vartheta_{2}+\Delta \varphi_{2}\right)}+\right. \\
\left.V_{3}\left(1+\varepsilon_{3}\right) e^{j\left(\vartheta_{3}+\Delta \varphi_{3}\right)}\right]
\end{gathered}
$$

where the $V_{i}$ and $\vartheta_{i}$ are the generic RMS values and the generic phase angles of the related phasors $\bar{V}_{i}$, respectively.

For the sake of simplicity, as first step of deeper study, a symmetrical three-phase system condition, can be assumed. This means that:

$$
V_{1}=V_{2}=V_{3}=V
$$

and

$$
\vartheta_{1}=0, \vartheta_{2}=\frac{2}{3} \pi \text { and } \vartheta_{3}=-\frac{2}{3} \pi
$$

By taking into account (4) and (5), (3) turns into:

$$
\begin{gathered}
\bar{V}_{R}=V\left[\left(1+\varepsilon_{1}\right) e^{j\left(0+\Delta \varphi_{1}\right)}+\left(1+\varepsilon_{2}\right) e^{j\left(\frac{2}{3} \pi+\Delta \varphi_{2}\right)}+\right. \\
\left.\left(1+\varepsilon_{3}\right) e^{j\left(-\frac{2}{3} \pi+\Delta \varphi_{3}\right)}\right]
\end{gathered}
$$

Eq. (6) relates the residual voltage to the contribution of uncertainty but more manipulations are needed to highlight how the module of $\bar{V}_{R}$ is affected by them.

To this purpose let us define $A_{i}$ as: 


$$
\begin{aligned}
& A_{1}=1+\varepsilon_{1} \\
& A_{2}=1+\varepsilon_{2} \\
& A_{3}=1+\varepsilon_{3}
\end{aligned}
$$

then, using the well-known Euler's formulas:

$$
\begin{gathered}
\bar{V}_{R}=V\left\{A_{1}\left[\cos \left(\Delta \varphi_{1}\right)+j \sin \left(\Delta \varphi_{1}\right)\right]+A_{2}\left[\operatorname { c o s } \left(\frac{2}{3} \pi+\right.\right.\right. \\
\left.\left.\Delta \varphi_{2}\right)+j \sin \left(\frac{2}{3} \pi+\Delta \varphi_{2}\right)\right]+A_{3}\left[\cos \left(-\frac{2}{3} \pi+\Delta \varphi_{3}\right)+\right. \\
\left.\left.j \sin \left(-\frac{2}{3} \pi+\Delta \varphi_{3}\right)\right]\right\}
\end{gathered}
$$

Through the application of the cosine and the sine of the sum of two angles:

$$
\begin{aligned}
& \cos (\alpha+\beta)=\cos \alpha \cos \beta-\sin \alpha \sin \beta \\
& \sin (\alpha+\beta)=\sin \alpha \cos \beta+\cos \alpha \sin \beta
\end{aligned}
$$

a further simplification of the residual voltage expression can be derived:

$$
\begin{array}{r}
\bar{V}_{R}=\frac{V}{3}\left\{A_{1} \cos \left(\Delta \varphi_{1}\right)+A_{2}\left[-\frac{1}{2} \cos \left(\Delta \varphi_{2}\right)-\right.\right. \\
\left.\frac{\sqrt{3}}{2} \sin \left(\Delta \varphi_{2}\right)\right]+A_{3}\left[-\frac{1}{2} \cos \left(\Delta \varphi_{3}\right)+\frac{\sqrt{3}}{2} \sin \left(\Delta \varphi_{3}\right)\right]+ \\
j\left\{A_{1} \sin \left(\Delta \varphi_{1}\right)+A_{2}\left[\frac{\sqrt{3}}{2} \cos \left(\Delta \varphi_{2}\right)-\frac{1}{2} \sin \left(\Delta \varphi_{2}\right)\right]+\right. \\
\left.\left.A_{3}\left[-\frac{\sqrt{3}}{2} \cos \left(\Delta \varphi_{3}\right)-\frac{1}{2} \sin \left(\Delta \varphi_{3}\right)\right]\right\}\right\}
\end{array}
$$

Now, at the light of the amplitudes of the $\Delta \varphi_{i}$ (by referring to Table I, which reports the limits of the accuracy classes as defined in [4]), two approximations can be done: $\cos \left(\Delta \varphi_{i}\right) \approx 1$ and $\sin \left(\Delta \varphi_{i}\right) \approx \Delta \varphi_{i}$. Hence:

$$
\begin{gathered}
\bar{V}_{R}=V\left\{A_{1}+A_{2}\left[-\frac{1}{2}-\frac{\sqrt{3}}{2} \Delta \varphi_{2}\right]+A_{3}\left[-\frac{1}{2}+\frac{\sqrt{3}}{2} \Delta \varphi_{3}\right]+\right. \\
\left.j\left\{A_{1} \Delta \varphi_{1}+A_{2}\left[\frac{\sqrt{3}}{2}-\frac{1}{2} \Delta \varphi_{2}\right]+A_{3}\left[-\frac{\sqrt{3}}{2}-\frac{1}{2} \Delta \varphi_{3}\right]\right\}\right\}
\end{gathered}
$$

At this point, the $A_{i}$ can be substituted into (12):

$$
\begin{array}{r}
\bar{V}_{R}=\frac{V}{3}\left\{1+\varepsilon_{1}-\frac{1}{2}-\frac{\sqrt{3}}{2} \Delta \varphi_{2}-\frac{1}{2} \varepsilon_{2}-\frac{\sqrt{3}}{2} \varepsilon_{2} \Delta \varphi_{2}-\frac{1}{2}+\right. \\
\frac{\sqrt{3}}{2} \Delta \varphi_{3}-\frac{1}{2} \varepsilon_{3}+\frac{\sqrt{3}}{2} \varepsilon_{3} \Delta \varphi_{3}+j\left\{\Delta \varphi_{1}+\varepsilon_{1} \Delta \varphi_{1}+\frac{\sqrt{3}}{2}-\frac{1}{2} \Delta \varphi_{2}+\right. \\
\left.\left.\frac{\sqrt{3}}{2} \varepsilon_{2}-\frac{1}{2} \varepsilon_{2} \Delta \varphi_{2}-\frac{\sqrt{3}}{2}-\frac{1}{2} \Delta \varphi_{3}-\frac{\sqrt{3}}{2} \varepsilon_{3}-\frac{1}{2} \varepsilon_{3} \Delta \varphi_{3}\right\}\right\}
\end{array}
$$

After some manipulations, the real and imaginary parts of (13) are:

$$
\begin{aligned}
& \text { Real }\left\{\bar{V}_{R}\right\}=V\left(\varepsilon_{1}-\frac{\sqrt{3}}{2} \Delta \varphi_{2}-\frac{1}{2} \varepsilon_{2}+\frac{\sqrt{3}}{2} \Delta \varphi_{3}-\frac{1}{2} \varepsilon_{3}\right) \\
& \operatorname{Im}\left\{\bar{V}_{R}\right\}=V\left(\Delta \varphi_{1}-\frac{1}{2} \Delta \varphi_{2}+\frac{\sqrt{3}}{2} \varepsilon_{2}-\frac{1}{2} \Delta \varphi_{3}-\frac{\sqrt{3}}{2} \varepsilon_{3}\right)
\end{aligned}
$$

The module of $\bar{V}_{R}$ is:

$$
\left|\bar{V}_{R}\right|=\sqrt{\left(\operatorname{Real}\left\{\bar{V}_{R}\right\}\right)^{2}+\left(\operatorname{Im}\left\{\bar{V}_{R}\right\}\right)^{2}}
$$

that combined with (14) and (15) turns into:
TABLE I. RATIO AND PHASE ERRORS LIMITS OF EACH ACCURACY CLASS

\begin{tabular}{|c|c|c|}
\hline $\begin{array}{c}\text { Accuracy } \\
\text { Class }\end{array}$ & $\begin{array}{c}\text { Ratio } \\
\text { Error } \\
\text { (\%) }\end{array}$ & $\begin{array}{c}\text { Phase } \\
\text { Error } \\
\text { (mrad) }\end{array}$ \\
\hline 0.1 & 0.1 & 1.5 \\
\hline 0.2 & 0.2 & 3 \\
\hline 0.5 & 0.5 & 6 \\
\hline
\end{tabular}

$$
\begin{gathered}
\left|\bar{V}_{R}\right|=V\left[\left(\varepsilon_{1}-\frac{\sqrt{3}}{2} \Delta \varphi_{2}-\frac{1}{2} \varepsilon_{2}+\frac{\sqrt{3}}{2} \Delta \varphi_{3}-\frac{1}{2} \varepsilon_{3}\right)^{2}+\right. \\
\left.\left(\Delta \varphi_{1}-\frac{1}{2} \Delta \varphi_{2}+\frac{\sqrt{3}}{2} \varepsilon_{2}-\frac{1}{2} \Delta \varphi_{3}-\frac{\sqrt{3}}{2} \varepsilon_{3}\right)^{2}\right]^{1 / 2}
\end{gathered}
$$

It can be noted that when all $\varepsilon_{i}$ and $\Delta \varphi_{i}$ are zero, (17) provides $\left|\bar{V}_{R}\right|=0$, according to the assumption of symmetrical voltages. Therefore, the expression between square brackets represents the error on $\bar{V}_{R}$ when a symmetric three-phase system of amplitude $V$ is considered. Of course, such error can be computed only if the values of the accuracy parameters $\varepsilon$ and $\Delta \varphi$ are already known, for each VT. Otherwise, (17) also allows to evaluate the uncertainty on $\bar{V}_{R}$ if $\varepsilon_{i}$ and $\Delta \varphi_{i}$ are treated as random variables and one of the methods, suggested by GUM [5] and its supplement 1 [6], is applied.

\section{RESEARCH OF THE MAXIMUM ERROR}

\section{A. Function study}

Eq. (17) provides a relationship between the errors of the VTs and that of the residual voltage. Therefore, it is interesting to determine which combination of $\varepsilon_{i}$ and $\Delta \varphi_{i}$ leads to the maximum error on $\bar{V}_{R}$.

As it is well-known, the derivative of a function must be studied to find the local maxima and minima. To this purpose, (17) is rewritten as:

$$
\begin{array}{r}
\left|\bar{V}_{R}\right|=V\left(\varepsilon_{1}{ }^{2}+\frac{3}{4} \Delta \varphi_{2}{ }^{2}+\frac{1}{4} \varepsilon_{2}{ }^{2}+\frac{3}{4} \Delta \varphi_{3}{ }^{2}+\frac{1}{4} \varepsilon_{3}{ }^{2}-\right. \\
\sqrt{3} \varepsilon_{1} \Delta \varphi_{2}-\varepsilon_{1} \varepsilon_{2}+\sqrt{3} \varepsilon_{1} \Delta \varphi_{3}-\varepsilon_{1} \varepsilon_{3}+\frac{\sqrt{3}}{2} \varepsilon_{2} \Delta \varphi_{2}- \\
\frac{3}{2} \Delta \varphi_{2} \Delta \varphi_{3}+\frac{\sqrt{3}}{2} \varepsilon_{3} \Delta \varphi_{2}-\frac{\sqrt{3}}{2} \varepsilon_{2} \Delta \varphi_{3}+\frac{1}{2} \varepsilon_{2} \varepsilon_{3}-\frac{\sqrt{3}}{2} \varepsilon_{3} \Delta \varphi_{3}+ \\
\Delta \varphi_{1}{ }^{2}+\frac{1}{4} \Delta \varphi_{2}{ }^{2}+\frac{3}{4} \varepsilon_{2}{ }^{2}+\frac{1}{4} \Delta \varphi_{3}{ }^{2}+\frac{3}{4} \varepsilon_{3}{ }^{2}-\Delta \varphi_{1} \Delta \varphi_{2}+ \\
\sqrt{3} \varepsilon_{2} \Delta \varphi_{1}-\Delta \varphi_{1} \Delta \varphi_{3}-\sqrt{3} \varepsilon_{3} \Delta \varphi_{1}-\frac{\sqrt{3}}{2} \varepsilon_{2} \Delta \varphi_{2}+\frac{1}{2} \Delta \varphi_{2} \Delta \varphi_{3}+ \\
\left.\frac{\sqrt{3}}{2} \varepsilon_{3} \Delta \varphi_{2}-\frac{\sqrt{3}}{2} \varepsilon_{2} \Delta \varphi_{3}-\frac{3}{2} \varepsilon_{2} \varepsilon_{3}+\frac{\sqrt{3}}{2} \varepsilon_{3} \Delta \varphi_{3}\right)^{1 / 2}
\end{array}
$$

Given that (18) is a 6 variables function, 6 partial derivatives must be computed and set equal to zero:

$$
\begin{array}{r}
\frac{\partial\left|\bar{V}_{R}\right|}{\partial \varepsilon_{1}}=0=2 \varepsilon_{1}-\varepsilon_{2}-\varepsilon_{3}-\sqrt{3} \Delta \varphi_{2}+\sqrt{3} \Delta \varphi_{3} \\
\frac{\partial\left|\bar{V}_{R}\right|}{\partial \varepsilon_{2}}=0=-\varepsilon_{1}+2 \varepsilon_{2}-\varepsilon_{3}+\sqrt{3} \Delta \varphi_{1}-\sqrt{3} \Delta \varphi_{3} \\
\frac{\partial\left|\bar{V}_{R}\right|}{\partial \varepsilon_{3}}=0=-\varepsilon_{1}-\varepsilon_{2}+2 \varepsilon_{3}-\sqrt{3} \Delta \varphi_{1}+\sqrt{3} \Delta \varphi_{2} \\
\frac{\partial\left|\bar{V}_{R}\right|}{\partial \Delta \varphi_{1}}=0=\sqrt{3} \varepsilon_{2}-\sqrt{3} \varepsilon_{3}+2 \Delta \varphi_{1}-\Delta \varphi_{2}-\Delta \varphi_{3} \\
\frac{\partial\left|\bar{V}_{R}\right|}{\partial \Delta \varphi_{2}}=0=-\sqrt{3} \varepsilon_{1}+\sqrt{3} \varepsilon_{3}-\Delta \varphi_{1}+2 \Delta \varphi_{2}-\Delta \varphi_{3}
\end{array}
$$




$$
\frac{\partial\left|\bar{V}_{R}\right|}{\partial \Delta \varphi_{3}}=0=\sqrt{3} \varepsilon_{1}-\sqrt{3} \varepsilon_{2}-\Delta \varphi_{1}-\Delta \varphi_{2}+2 \Delta \varphi_{3}
$$

The solution of the system of equations (19) provides the combination of $\varepsilon_{i}$ and $\Delta \varphi_{i}$ for which (17) (or (18)) takes its maximum (or minimum). Unfortunately, only 2 out of the 6 equations are linearly independent. This means that (19) has $\infty^{4}$ solutions. Two of the linearly independent equations are, for example:

$$
\begin{aligned}
& \varepsilon_{1}-\varepsilon_{3}+m \Delta \varphi_{1}-l \Delta \varphi_{2}+m \Delta \varphi_{3} \\
& \varepsilon_{2}-\varepsilon_{3}+l \Delta \varphi_{1}-m \Delta \varphi_{2}-m \Delta \varphi_{3}
\end{aligned}
$$

where $m=0.57735$ and $l=1.1547$.

\section{B. Maximum Error algorithm}

To overcome the aforementioned infinite solutions issue, a numerical approach has been implemented. In particular, it consists in the application of the following steps:

1. two variables among the six are chosen as dependent, for example $\varepsilon_{1}$ and $\varepsilon_{2}$;

2. the remaining variables $\varepsilon_{3}, \Delta \varphi_{1}, \Delta \varphi_{2}$ and $\Delta \varphi_{3}$, are varied within the limits of a given accuracy class with a certain resolution; in particular:

a. 0.5-accuracy class has been chosen because it contains all the limits of the other classes (Table I).

b. steps of $0.5 \mathrm{mrad}$ for the phase and $0.01 \%$ for the ratio have been chosen.

3. in each iteration $\varepsilon_{1}$ and $\varepsilon_{2}$ have been computed according to (20) and (21). The solution is discarded if at least one of them falls outside the limits of 0.5 -accuracy class. Therefore, when it is greater than $0.5 \%$ or lower than $0.5 \%$.

4. The value of, $\left|\bar{V}_{R}\right|$ is computed on the basis of the solutions obtained in the previous steps.

5. At the end of all the iterations (1578126 for the considered limits and resolution), the maximum value of $\left|\bar{V}_{R}\right|$ can be easily detected.

\section{Results}

Fig. 2 shows the plot of $\left|\bar{V}_{R}\right|$ values obtained in all the iterations for which a correct solution of (19) (i.e. conditions stated in previous step 3 are fulfilled) occurs. It can be observed that there are two combinations for which $\left|\bar{V}_{R}\right|$ takes the highest value: about $1 \%$. The values of the parameters $\varepsilon_{i}$ and $\Delta \varphi_{i}$ corresponding to such peaks are shown in Table II.

It arises that the accuracy class of the LPVTs used in a threephase system strongly affect the error affecting the value of $\left|\bar{V}_{R}\right|$ and there is not a unique condition and relationship between the ratio and phase errors of the LPVT and the error affecting $\left|\bar{V}_{R}\right|$.

The new Standard on LPVTs [7] reports a table of accuracy classes of LPVTs used for special applications (like, in particular, for the residual voltage evaluation). However, it does not report information and instructions regarding the correlation of accuracy class and uncertainty on $\left|\bar{V}_{R}\right|$. In this connection, the analysis performed in the follows may be of help.

\section{UNCERTAINTY EVALUATION}

As mentioned in Section II, the residual voltage error can be obtained only if the values of the accuracy parameters $\varepsilon$ and $\Delta \varphi$

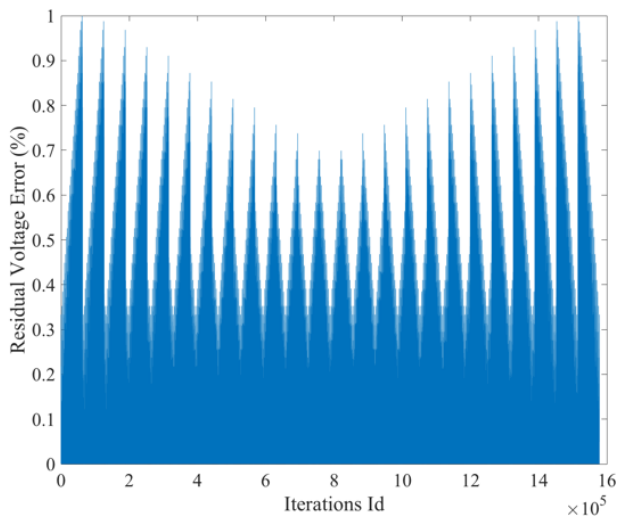

Fig. 2. Graph of the residual voltage when all iterations are applied

TABLE II. VALUES OF THE SIX PARAMETERS CORRESPONDING TO THE PEAK OF THE FUNCTION $\left|\bar{V}_{R}\right|$

\begin{tabular}{|c|c|c|c|c|c|}
\hline$\varepsilon_{\mathbf{1}}$ & $\boldsymbol{\varepsilon}_{\mathbf{2}}$ & $\boldsymbol{\varepsilon}_{\mathbf{3}}$ & $\begin{array}{c}\Delta \boldsymbol{\varphi}_{\mathbf{1}} \\
(\mathbf{m r a d})\end{array}$ & $\begin{array}{c}\Delta \boldsymbol{\varphi}_{\mathbf{2}} \\
(\mathbf{m r a d})\end{array}$ & $\begin{array}{c}\Delta \boldsymbol{\varphi}_{\mathbf{3}} \\
(\mathbf{m r a d})\end{array}$ \\
\hline 0.499 & 0.460 & 0.460 & 0 & 6 & -6 \\
\hline-0.499 & -0.460 & -0.460 & 0 & -6 & 6 \\
\hline
\end{tabular}

are already known, for each VT. However, in practical situations, this is not possible and the uncertainty affecting $\left|\bar{V}_{R}\right|$ must be determined. The GUM and its Supplement 1 provide detailed explanation on how estimating such value, but the implementation of the methods they provided may be quite complex and certainty not ease for technicians who are not metrologist or university professors. For this reason, in this Section, a simplified expression for the uncertainty evaluation of the residual voltage measurement is presented. The aim consists in obtaining an easy-to use formula directly applicable in field, when only the accuracy class of the VTs is known.

\section{A. Uncertainty formula}

To obtain the aformentioned expression let's start from (17), which expresses the residual voltage module considering all the ratio and phase errors contributes of the VTs. Defining:

$$
\begin{aligned}
& X=\varepsilon_{1}-\frac{\sqrt{3}}{2} \Delta \varphi_{2}-\frac{1}{2} \varepsilon_{2}+\frac{\sqrt{3}}{2} \Delta \varphi_{3}-\frac{1}{2} \varepsilon_{3} \\
& Y=\Delta \varphi_{1}-\frac{1}{2} \Delta \varphi_{2}+\frac{\sqrt{3}}{2} \varepsilon_{2}-\frac{1}{2} \Delta \varphi_{3}-\frac{\sqrt{3}}{2} \varepsilon_{3}
\end{aligned}
$$

( $X$ and $Y$ represents, according to (17), the p.u. real and imaginary part of $\left|\bar{V}_{R}\right|$, respectively) eq. (17) can be written symbolically as:

$$
Z=\sqrt{X^{2}+Y^{2}}
$$

where the voltage $V$ from (17) has been intentionally omitted to obtain a per-unit expression. In this form, if $X$ and $Y$ are random variable with given characteristcs, (24) represents a well-known probability density function: the Rayleigh distribution, pending $X$ and $Y$ are indipendent random variables having the same gaussian distribution $N\left(0, \sigma^{2}\right)$. 
Let's now consider (22) and (23). Both of them are a linear combination of four variables and it is well known that, given a random variable $f$ defined as:

$$
f=a g+b h,
$$

where $g$ and $h$ are generic random variables and $a$ and $b$ are numerical coefficients, its variance $\sigma_{f}^{2}$ is given by:

$$
\sigma_{f}^{2}=a^{2} \sigma_{g}^{2}+b^{2} \sigma_{h}^{2}
$$

where $\sigma_{g}^{2}$ and $\sigma_{h}^{2}$ are the variances of $g$ and $h$, respectively.

Therefore, applied to our case, this leads to:

$$
\begin{aligned}
& \sigma_{X}^{2}=\sigma_{\varepsilon 1}^{2}+\frac{3}{4} \sigma_{\Delta \varphi 2}^{2}+\frac{1}{4} \sigma_{\varepsilon 2}^{2}+\frac{3}{4} \sigma_{\Delta \varphi 3}^{2}+\frac{1}{4} \sigma_{\varepsilon 3}^{2} \\
& \sigma_{Y}^{2}=\sigma_{\Delta \varphi 1}^{2}+\frac{1}{4} \sigma_{\Delta \varphi 2}^{2}+\frac{3}{4} \sigma_{\varepsilon 2}^{2}+\frac{1}{4} \sigma_{\Delta \varphi 3}^{2}+\frac{3}{4} \sigma_{\varepsilon 3}^{2}
\end{aligned}
$$

Another important simplification can be obtained if a realistic assumption is considered: 3 VTs of the same accuracy class are adopted in the 3-phase system voltage measurement. Such a statement is not unrealistic, in fact there is no reason to adopt 3 different accuracy class voltage transformers for the measurement of 3 voltages of the same line. At the light of this (27) and (28) turns into:

$$
\begin{aligned}
& \sigma_{X}^{2}=\sigma_{\varepsilon}^{2}+\frac{3}{4} \sigma_{\Delta \varphi}^{2}+\frac{1}{4} \sigma_{\varepsilon}^{2}+\frac{3}{4} \sigma_{\Delta \varphi}^{2}+\frac{1}{4} \sigma_{\varepsilon}^{2} \\
& \sigma_{Y}^{2}=\sigma_{\Delta \varphi}^{2}+\frac{1}{4} \sigma_{\Delta \varphi}^{2}+\frac{3}{4} \sigma_{\varepsilon}^{2}+\frac{1}{4} \sigma_{\Delta \varphi}^{2}+\frac{3}{4} \sigma_{\varepsilon}^{2}
\end{aligned}
$$

hence:

$$
\begin{gathered}
\sigma_{X}^{2}=\frac{3}{2}\left(\sigma_{\Delta \varphi}^{2}+\sigma_{\varepsilon}^{2}\right) \\
\sigma_{Y}^{2}=\frac{3}{2}\left(\sigma_{\Delta \varphi}^{2}+\sigma_{\varepsilon}^{2}\right) \\
\sigma_{X}^{2}=\sigma_{Y}^{2}
\end{gathered}
$$

where the notation assumed is the same as for the generic case presented in (25) and (26). From (31) and (32) its clear that $X$ and $Y$ have the same the variance.

Moreover, according to the Central Limit Theorem, $X$ and $Y$ as defined by (22) and (23) can be considered as having normal distribution given that they are linear combination of random variables.

These result leads to three conclusions relevant to the Rayleigh distribution:

- $\quad$ it has been demonstrated that the two terms $X$ and $Y$ of the residual voltage module have the same distribution, hence in accordance with Rayleigh conditions;

- $\quad X$ and $Y$ are Normal;

- if the two variances are identical, the two random variables $X$ and $Y$ are strongly correlated, hence not indipendent as requested in the Rayleigh distribution.

However, assuming for a moment that the two random variables are indipendent, the Rayleigh variance expression can be adopted:

$$
\sigma_{R}^{2}=\left(2-\frac{\pi}{2}\right) \sigma^{2}
$$

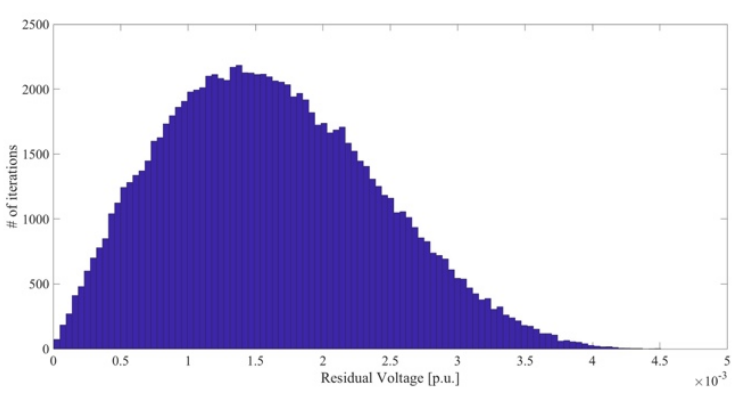

Fig. 3. Pdf of the residual voltage when 3 VTs of 0.1 accuracy class are considered

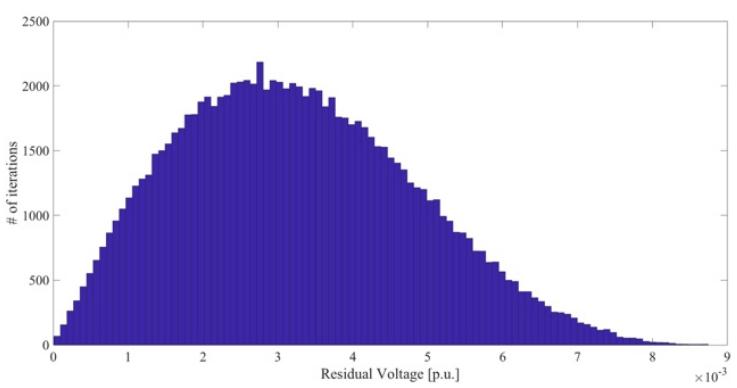

Fig. 4. Pdf of the residual voltage when 3 VTs of 0.2 accuracy class are considered

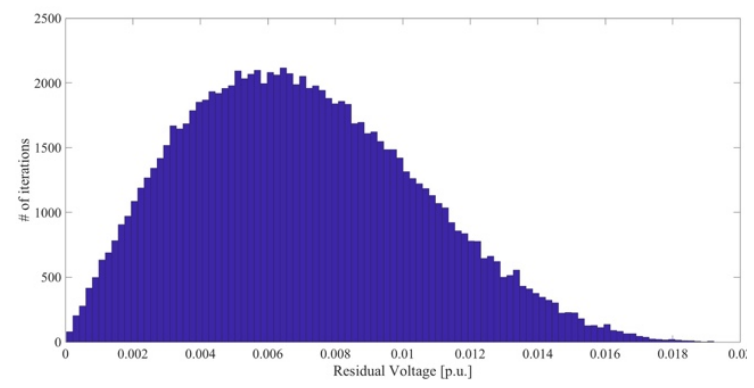

Fig. 5. Pdf of the residual voltage when 3 VTs of 0.5 accuracy class are considered

In the following subsection, some results are presented in order to prove the effectiveness of such a formula despite from the fact that the variables of interest are not indipendent.

\section{B. Monte Carlo results.}

First of all, let's consider the 6 variables contained in (17) as random variables uniformely distributed with zero mean. Then, 1 million Monte Carlo trials are computed to obtain the pdf and the variance of $\left|\bar{V}_{R}\right|, X$ and $Y$. As for $\left|\bar{V}_{R}\right|$, fig 3, 4 and 5 show its pdfs in the case of three VTs with $0.1,0.2$ and 0.5 accuracy class, respectively. Moreover, the pdfs of $X$ and $Y$ are presented in fig. 6 and 7, only for the 0.1 accuracy class case for the sake of brevity. From these two figures, it can be higligthed that the linear combination of more than two uniformly distributed random variables turns into a gaussian distribution with zero mean as known from the literature. Hence, $X$ and $Y$ can be used to satisfy the Rayleigh condition.

At this point, the variances of $\left|\bar{V}_{R}\right|$, one calculated applying $\mathrm{MC}$ to (17), $\sigma_{V r}^{2}$, and one calculated using the variance of the 
Rayleigh distribution (34), $\sigma_{R}^{2}$, can be compared. The results of the comparison are listed in Table III for the $0.1,0.2$ and 0.5 accuracy class cases, where the standard deviations $\sigma_{V r}$ and $\sigma_{R}$ (that is the standard uncertainties) are shown. As it can be seen, despite the fact that $X$ and $Y$ are not indipendent, the variances (and the standard deviations) are almost identical for all the considered accuracy classes: they differ for the second digit only. This allows to conclude that the proposed simplified expression can be usefully employed to estimate the uncertainty on $\left|\bar{V}_{R}\right|$.

\section{CONCLUSIONS}

The operation of Smart Grids in presence of Distributed Energy Resources has required the introduction of new technologies in power systems, like digital communication, new type of switchgears, different network topologies (like Ring Main Units, redundant radial networks etc.) and the massive us of LPIT, which are replacing the conventional Inductive IT.

The use of LPITs has allowed to implement many new operating and measurement functions due to their better performance with respect to inductive ITs (bandwidth, accuracy, linearity, etc.). However, one critical value, still widely used for the diagnostic and the operation of the power networks, namely the residual voltage, requires that LPVTs feature higher accuracies with respect to those of ITs with open triangle. The correlation of the accuracy class of LPVTs to the uncertainty affecting the residual voltage is not straightforward. However, this relationship is strongly demanded by practitioners and all people involved in network design and operation.

For all this, the presented paper has reported the theoretical study and the results of simulations performed in order to demonstrate the effects of the accuracy class of the LPIT on the residual voltage uncertainty. The assumption assumed in the paper concern the analysis of a symmetrical 3-phase system. However, future works must investigate the same effects when such condition is not satisfied.

The aim of this study has been to put all such operators in a condition to simply evaluate and know which is the expected uncertainty affecting the residual voltage when employing LPVTs with a given accuracy class. To this purpose, a simplified expression based on the Rayleigh distribution has been derived. Results demonstrated the effectiveness of this assumption, even if a particular request of the Rayleigh distribution was not satisfied: the independency of the two variables $X$ and $Y$. Such important supposition requires further studies, exploring more cases to prove its success.

\section{REFERENCES}

[1] IEC 61850-9-2:2011, "Communication networks and systems for power utility automation - Part 9-2: Specific communication service mapping (SCSM) - Sampled values over ISO/IEC 8802-3", International Standardization Organization, Geneva, Switzerland, 2008.

[2] IEC 61869-1:2011, "Instrument transformers - Part 1: General requirements", International Standardization Organization, Geneva, Switzerland, 2011.

[3] IEC 61869-2:2011, "Instrument transformers - Part 2: Additional requirements for current transformers", International Standardization Organization, Geneva, Switzerland, 2011.

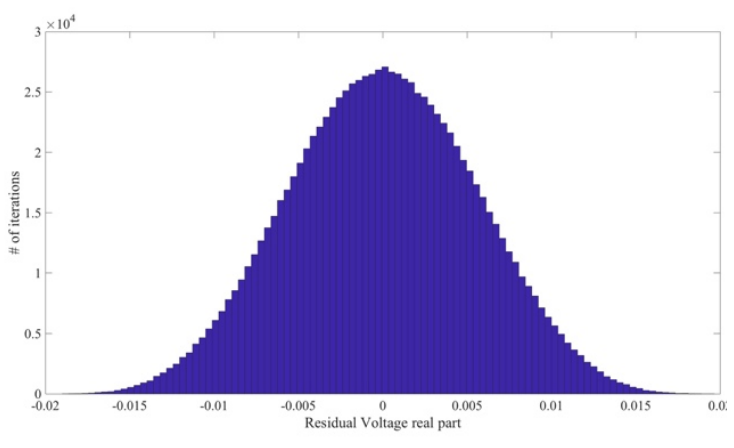

Fig. 6. Pdf of $X$, the real part and the first term of (17), when the 3 VTs have 0.5 accuracy class

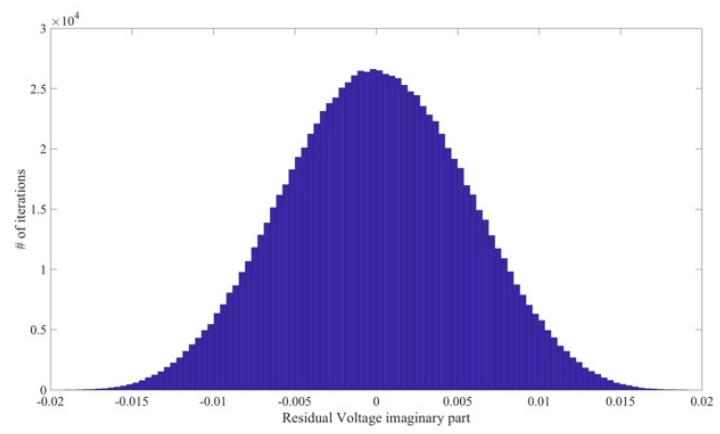

Fig. 7. Pdf of $Y$, the imaginary part and the second term of (17), when the 3 VTs have 0.5 accuracy class

TABLE III. VARIANCES AND STANDARD DEVIATIONS OF $\left|\bar{V}_{R}\right|$, CALCULATED WITH MONTE CARLO OR CALCULATED WITH THE RAYLEIGH FORMULA

\begin{tabular}{|c|c|c|c|c|}
\hline Accuracy class & $\boldsymbol{\sigma}_{\mathbf{V r}}^{\mathbf{2}}$ & $\boldsymbol{\sigma}_{\mathbf{R}}^{\mathbf{2}}$ & $\boldsymbol{\sigma}_{\mathbf{V r}}$ & $\boldsymbol{\sigma}_{\mathbf{R}}$ \\
\hline 0.1 & $6.1 \mathrm{e}-7$ & $6.9 \mathrm{e}-7$ & $7.8 \mathrm{e}-4$ & $8.3 \mathrm{e}-4$ \\
\hline 0.2 & $2.5 \mathrm{e}-6$ & $2.8 \mathrm{e}-6$ & $1.6 \mathrm{e}-3$ & $1.7 \mathrm{e}-3$ \\
\hline 0.5 & $1.2 \mathrm{e}-5$ & $1.3 \mathrm{e}-5$ & $3.5 \mathrm{e}-3$ & $3.6 \mathrm{e}-3$ \\
\hline
\end{tabular}

[4] IEC 61869-3:2011, "Instrument transformers - Part 3: Additional requirements for inductive voltage transformers", International Standardization Organization, Geneva, Switzerland.

[5] ISO/IEC Guide 98-3:2008, "Uncertainty of measurement --Part 3: Guide to the expression of uncertainty in measurement (GUM:1995)", International Standardization Organization, Geèneva, Switzerland, 2008.

[6] ISO/IEC Guide 98-3/Suppl.1:2008, "Evaluation of measurement data Supplement 1 to the "Guide to the expression of uncertainty in measurement" - Propagation of distributions using a Monte Carlo method", International Standardization Organization, Geneva, Switzerland 2008

[7] IEC 61869-11:2017, "Instrument transformers - Part 2: Additional requirements for current transformers", International Standardization Organization, Geneva, Switzerland, in press.

[8] IEC 61869-105, "Uncertainty in the calibration of Instruemnt Transformers", under preparation by IEC TC38/WG55.

[9] IEC 60050:2017, "International Electrothecnical Vocabulary (IEV)", 2017. 